\title{
Continuous Cropping and Natural Fallow Practices Affect Tobacco Fitness and Soil Microbiomes
}

\author{
Hao Jiang ${ }^{1}$, Huanhuan Shao ${ }^{2}$, Quanju Xiang ${ }^{3}$, Yunfu $\mathrm{Gu}^{3}$, Bin $\mathrm{Li}^{4}$, Huizhan $\mathrm{Gu}^{4}, \mathrm{Yu}$ \\ Guan $^{4}$, and Yuanbin Zhang ${ }^{1}$ \\ ${ }^{1}$ Institute of Mountain Hazards and Environment Chinese Academy of Sciences \\ ${ }^{2}$ Sichuan Normal University \\ ${ }^{3}$ Sichuan Agricultural University \\ ${ }^{4}$ Sichuan Branch of Chinese National Tobacco Corporation
}

October 15,2020

\begin{abstract}
Natural fallow practice has been identified as an effective way to overcome obstacles of continuous cropping. However, how the resulting soil microbial changes impact plant fitness, and how the context-specific differences diverge from those caused by continuous cropping remain largely unknown. This study used the third-year continuous tobacco cropping soil (CCS) and natural fallow soil (FS) to cultivate Nicotiana tabacum. The influences of soil microorganisms on the fitness of N. tabacum were assessed by reassembling soil microbial communities. Then, the bacterial and fungal community assembly of the bulk soil and the rhizosphere were characterized using amplicon sequencing and statistical analysis. The results indicated that soil microorganisms play more important roles for plant fitness for N. tabacum grown in FS compared with CCS. Moreover, the abiotic context of FS exerts stronger effects compared with those of CCS for the reassembly of soil microbiomes. Comparative analysis identified the context-specific microbial clades and the differential strength of rhizosphere effects. In conclusion, this paper provides context-specific microbial evidence, which may unravel the potential mechanism underlying the different response of N. tabacum to changes of soil microbiomes induced by natural fallow and continuous cropping practices.
\end{abstract}

Submitted to Land Degradation 83 Development

Continuous Cropping and Natural Fallow Practices Affect Tobacco Fitness and Soil Microbiomes

Hao Jiang ${ }^{1}$, Huanhuan Shao ${ }^{2}$, Quanju Xiang ${ }^{3}$, Yunfu $\mathrm{Gu}^{3}$,

Bin $\mathrm{Li}^{4}$, Huizhan $\mathrm{Gu}^{4}$, Yu Guan ${ }^{4}$, Yuanbin Zhang ${ }^{1}$

${ }^{1}$ Institute of Mountain Hazards and Environment, Chinese Academy of Sciences, Chengdu 610041, China

${ }^{2}$ College of Life Sciences, Sichuan Normal University, No. 1819, Section 2, Chenglong Avenue, Longquan District, Chengdu, 610101, China

3 Department of Microbiology, College of Resources, Sichuan Agricultural University, Chengdu, 611130, China

${ }^{4}$ Sichuan Branch of China National Tobacco Corporation, Chengdu 610000, China

Correspondence address:

Yuanbin Zhang 
Institute of Mountain Hazards and Environment, Chinese Academy of Sciences

Chengdu 610041, China

Tel: +86-28-85557542; Fax: +86-28-85222258; E-mail: zhangyb@imde.ac.cn

Running title:Tobacco fitness and soil microbiomes

\section{ABSTRACT}

Natural fallow practice has been identified as an effective way to overcome obstacles of continuous cropping. However, how the resulting soil microbial changes impact plant fitness, and how the context-specific differences diverge from those caused by continuous cropping remain largely unknown. This study used the third-year continuous tobacco cropping soil (CCS) and natural fallow soil (FS) to cultivateNicotiana tabacum. The influences of soil microorganisms on the fitness of $N$. tabacum were assessed by reassembling soil microbial communities. Then, the bacterial and fungal community assembly of the bulk soil and the rhizosphere were characterized using amplicon sequencing and statistical analysis. The results indicated that soil microorganisms play more important roles for plant fitness for $N$. tabacum grown in FS compared with CCS. Moreover, the abiotic context of FS exerts stronger effects compared with those of CCS for the reassembly of soil microbiomes. Comparative analysis identified the context-specific microbial clades and the differential strength of rhizosphere effects. In conclusion, this paper provides context-specific microbial evidence, which may unravel the potential mechanism underlying the different response of $N$. tabacum to changes of soil microbiomes induced by natural fallow and continuous cropping practices.

KEYWORDS: continuous cropping, plant fitness, natural fallow, rhizosphere effect, microbial microbiome

\section{INTRODUCTION}

Climate change, human activities, and environmental pollution has resulted in a series of adverse impacts, such as land degradation and a shortage of agriculturally productive land, thus putting agricultural production at risk. To achieve stable yields and continuous benefits, a number of field- or cash-crops are often continuously cropped, which is combined with the intensive application of fertilizers by producers. As a result, obstacles to continuous cropping occur frequently, such as disruptions of soil health, soil microbial community composition, and function (Garbeva et al., 2004). These effects are often responsible for soilborne disease enrichment (Berendsen et al., 2012). However, a number of species may be able to cope with successive changes of the soil microenvironment via physiological adaptation (Teste et al., 2017; Hu et al., 2018). It has been postulated that beneficial plant-microbe interactions might offer promising strategies (Berg, 2009; Berendsen et al., 2012). An increasing body of evidence confirmed that beneficial microbes can optimize plant growth (Venkatachalam et al., 2014) and protect roots against microbial pathogens (Bonanomi et al., 2018). For example, the flavobacterium TRM1, found in the rhizosphere of species resistant to the soil-borne pathogen Ralstonia solanacearum could suppress disease development and enhance wilt resistance in tomato (Kwak et al., 2018). Bacterial root commensals have been suggested to shape fungal and oomycetal community structure and protect plants against fungi and oomycetes, thus promoting plant survival (Duran et al., 2018). However, knowledge of the microorganisms underlying the process and the identity of beneficial microbes still remains unclear.

Soil microorganisms have been confirmed to be involved in many key processes of the soil ecosystem (Jansson \& Hofmockel, 2020) and play key roles in regulating soil carbon dynamics (Zhou et al., 2012), nutrition cycling (Kuypers et al., 2018; Luo et al., 2018), and the turnover of organic matter (Beulig et al., 2016; Xun et al., 2018). Still, producers tend to ignore the potential risks arising from changing soil microorganisms and focus more on specific agricultural management practices (e.g., long-term monoculture and fertilizer utilization) to achieve high yields. With regard to this situation, several productive and successful agricultural practices, such as organic farming (Reganold \& Wachter, 2016), no-tillage (Ashworth et al., 2017), and rotation (Liu et al., 2019), have been applied and many crucial findings based on soil microbiomes have emerged (Wang et al., 2017; Chen et al., 2018; Hartman et al., 2018). However, the current shortage of labor seriously limits these applications. Instead, conventional practices, such as natural fallow, are being 
widely used worldwide. For most crop plants, however, knowledge on the changes of microbial communities under continuous cropping and natural fallow conditions remains scarce. Moreover, the major contributors, particularly those in rhizosphere microbiomes, to plant growth and soil health remain equally elusive (Lau \& Lennon, 2012; Venkatachalam et al., 2014; Bender et al., 2016).

Nicotiana tabacum is a model species of agricultural biotechnology and is very sensitive to continuous cropping. This study hypothesized that the changes of soil microbial community, especially changes of the specific microbial clades and rhizosphere effects caused by the natural fallow practice, could enhance plant fitness. To test our hypothesis, continuous tobacco cropping soil (the third year) and natural fallow soil were used to cultivate $N$. tabacumplants. First, the influences of soil microorganisms of both conditioned soils on $N$. tabacum fitness were assessed by reassembling soil microbial communities. Then, the bacterial and fungal community assembly of the bulk soil and rhizosphere were characterized. The aims of the study were (i) to identify whether changes of the soil microbiome, induced by natural fallow practice, support a more diverse and active soil biota compared with changes induced by continuous cropping practice, and would consequently enhance $N$. tabacum fitness; and (ii) to identify the context-specific differences of microbiomes and rhizosphere effects caused by continuous tobacco cropping and natural fallow practices.

\section{MATERIALS AND METHODS}

\subsection{Experimental design}

To investigate the effects of microorganisms on $N$. tabacumgrowth, all plant-growth experiments were conducted in a greenhouse under natural light and ambient conditions. The daytime temperature was $16-33^{\circ} \mathrm{C}$, the night-time temperature was $11-20^{\circ} \mathrm{C}$, and the relative humidity was $40-85 \%$ during the treatment period. The experiments were conducted at the Pu'an Flue-cured Tobacco Scientific Research Base, in Guangyuan, Sichuan Province, China (32.004N, 105.493E). Briefly, after homogenization, natural field-sampled soil was divided into two parts in March 2017. The properties of the soil were as follows (based on $\mathrm{kg}^{-1} \mathrm{dry}$ soil): $\mathrm{pH} 7.6$, organic carbon $9.68 \mathrm{~g}$, total nitrogen $1.62 \mathrm{~g}$, total phosphorus $0.58 \mathrm{~g}$, and total potassium $18.72 \mathrm{~g}$. One part was used to cultivate N. tabacum for two consecutive seasons from April 2017 to September 2018, which was defined as continuous cropping soil (CCS). The other part remained uncultivated and was defined as natural fallow soil (FS). In 2019, half of each conditioned soil was sterilized. This procedure results in four different types of conditioned soil: (i) Continuous cropping soil without sterilization treatment (C_NS); (ii) continuous cropping soil with sterilization treatment (C_S); (iii) natural fallow soil without sterilization treatment (F_NS); and (iv) natural fallow soil with sterilization treatment (F_S). Therefore, the experimental layout was completely randomized with two factors (soil type and sterilization treatment). Three replicates with three plants each were used to account for sampling errors. $N$. tabacum seeds were first imbibed in sterile water for $24 \mathrm{~h}$, and then surface-sterilized in $75 \%$ ethanol for $30 \mathrm{~s}$, followed by a brief wash with $10 \%$ $\mathrm{NaClO}(30 \mathrm{~min})$ and five subsequent washes with sterile water. In the end, $N$. tabacumseeds were sown onto the surface of each conditioned soil type. Nitrogen fertilizer $\left(105 \mathrm{~kg} \mathrm{ha}^{-1}\right)$ was applied to ensure good $N$. tabacumgrowth.

\subsection{Sample collection and DNA extraction}

N. tabacum plants were harvested at flowering stage in July 2019 and were separated into leaves, stems, roots, flowers, and seeds. Biomass samples were separately oven-dried $\left(70^{\circ} \mathrm{C}\right.$ for $\left.48 \mathrm{~h}\right)$ to constant weight. Leaf dry mass per unit area (LMA, $\mathrm{g} \mathrm{m}^{-2}$ ) was calculated as the quotient of the mass and the area of the leaf sample. For microbiome analysis (Ortas, 1997), 24 soil samples were collected (two conditioned soil (continuous cropping soil vs. natural fallow soil) $\mathrm{x}$ two treatments (sterilized vs. non-sterilized) $\mathrm{x}$ two sample types (bulk soil and rhizosphere) x three replicates). Soil particles not in contact with the N. tabacum root system or other visible plants were collected and defined as bulk soil. In addition, soil remaining on the root segments after a strong shake was considered to be rhizosphere soil. Fresh root $(10 \mathrm{~g})$ was added to $90 \mathrm{ml}$ sterilized water, followed by $10 \mathrm{~min}$ ultra-sonication and $30 \mathrm{~min}$ shaking at $200 \mathrm{rpm}$. Rhizosphere soil from this suspension was collected by centrifugation at $12000 \mathrm{x}$ g for $15 \mathrm{~min}$ at $25 \mathrm{degC}$. Both bulk soil and rhizosphere soil were stored at $-20 \operatorname{deg} C$ until DNA extraction. Each of three biological replicates 
pooled three individuals. Approximately $250 \mathrm{mg}$ of bulk soil and rhizosphere soil were used for each DNA extraction. DNA extraction was performed with the E.Z.N.A.(r) Soil DNA Kit (Omega Bio-Tek, Norcross, GA, USA) following the manufacturer's instructions. The DNA extract was assessed on a $1 \%$ agarose gel, and DNA concentration and purity were determined with a NanoDrop 2000 UV-vis spectrophotometer (Thermo Scientific, Wilmington, DE, USA).

\subsection{PCR, library preparation and sequencing}

The hypervariable region V3-V4 of the bacterial 16S rRNA gene was amplified with the PCR primer pairs 338F (5'-ACTCCTACGGGAGGCAGCAG-3') and 806R (5'-GGACTACHVGGGTWTCTAAT3') (Mori et al., 2014). The fungal ITS1 region was amplified using PCR primers ITS1F (5'CTTGGTCATTTAGAGGAAGTAA-3') and ITS2R (5'-GCTGCGTTCTTCATCGATGC-3') (Adams et al., 2013). PCR amplification was performed as follows: initial denaturation at $95 \operatorname{deg}$ for $3 \mathrm{~min}$, followed by 27 (338F_806R) / 35 (ITS1F_ITS2R) cycles of denaturation at $95 \operatorname{degC}$ for $30 \mathrm{~s}$, annealing at $55 \operatorname{deg}$ for $30 \mathrm{~s}$, extension at $72 \operatorname{deg} \mathrm{C}$ for $45 \mathrm{~s}$, single extension at $72 \mathrm{deg} \mathrm{C}$ for $10 \mathrm{~min}$, and termination at $4 \mathrm{degC}$. The PCR mixtures contained $5 \times$ TransStart FastPfu buffer $(4 \mu \mathrm{L}), 2.5 \mathrm{mM}$ dNTPs $(2 \mu \mathrm{L})$, forward primer $(5$ $\mu \mathrm{M} ; 0.8 \mu \mathrm{L})$, reverse primer $(5 \mu \mathrm{M} ; 0.8 \mu \mathrm{L})$, TransStart FastPfu DNA Polymerase $(0.4 \mu \mathrm{L})$, bovine serum albumin (BSA; $0.2 \mu \mathrm{L}$ ), template DNA (10 ng), and up to $20 \mu \mathrm{L} \mathrm{ddH}_{2} \mathrm{O}$. PCR reactions were performed in triplicate. The PCR products were extracted from $2 \%$ agarose gel and purified using the AxyPrep DNA Gel Extraction Kit (Axygen Biosciences, Union City, CA, USA) according to manufacturer's instructions and were quantified using Quantus Fluorometer (Promega, Madison, WI, USA).

Purified amplicons were pooled equimolar and were paired-end sequenced $(2 \times 300)$ on an Illumina MiSeq platform (Illumina, San Diego, CA, USA) by Majorbio Bio-Pharm Technology Co., Ltd. (Shanghai, China). The raw reads were deposited into the NCBI Sequence Read Archive (SRA) database under Accession Number: PRJNA609016.

\subsection{Sequence processing}

Raw fastq files were quality-filtered by Trimmomatic (Bolger et al., 2014) and merged by FLASH (Magoc \& Salzberg, 2011) applying the following criteria: (i) Reads were truncated at any site receiving an average quality score $<20$ over a 50-bp sliding window. (ii) Sequences with overlap longer than 10 bp were merged according to their overlap with mismatch of no more than $2 \mathrm{bp}$. (iii) Sequences of each sample were separated according to barcodes (exact matching) and primers (allowing a 2-nucleotide mismatch). Reads containing ambiguous bases were removed. Operational taxonomic units (OTUs) were clustered with a $97 \%$ similarity cutoff using UPARSE (Edgar, 2013). The taxonomy of each 16S rRNA gene sequence was analyzed by the RDP classifier algorithm (http://rdp.cme.msu.edu/) against the Silva database (Quast et al., 2013), using a confidence threshold of $70 \%$ and implementation in QIIME (Caporaso et al., 2010). ITS sequences were processed similarly and taxonomy was assigned using the UNITE database (https://unite.ut.ee/index.php) with the RDP classifier in QIIME.

\subsection{Statistical analysis}

Physiological data were analyzed using the SPSS 17.0 software (SPSS Inc., Chicago, IL, USA). Significant differences among the means of different treatments were determined by Tukey's multiple range tests after conducting tests of homogeneity for variances. Differences were considered statistically significant at the $p$ $<0.05$ level. To assess alpha-diversity, communities were rarified to 11000 (bacteria) and 10000 (fungi) sequences per sample. Alpha-diversity indexes (Ace index and Sobs index) were calculated using QIIME (Caporaso et al., 2010). The significances of differences among treatments were assessed using the Welch's ttest. Similarities and differences arising from microbiomes of different soil samples were visualized with Circos (Krzywinski et al., 2009). Bray-Curtis distances between samples were used for principal coordinate analysis (PCoA) to assess the major variance components of the beta-diversity. Permutational multivariate analysis of variance (PERMANOVA) was used to evaluate group differences. Discriminant taxa were significantly retrieved by linear discriminant analysis (LDA) effect size (LEfSe) for bulk-soil and rhizosphere-microbial communities (Segata et al., 2011). 


\section{RESULTS}

\subsection{Effects of soil microorganisms on $N$. tabacumfitness}

Variation and development of soil microbiomes exert distinct effects on $N$. tabacumfitness. As shown in Figure 1a, soil sterilization treatment prior to sowing significantly decreased biomass accumulation when plants grew in FS $(p<0.05)$, but not if grown in CCS $(p=0.94)$. In addition, no significant difference was found in biomass allocation patterns in $N$. tabacum, with one exception: soil sterilization treatment prior to sowing markedly decreased leaf mass when plants were grown in FS (Figure 1b). Specifically, the LMA of N. tabacum plants grown in F_S were significantly higher than those grown in F_NS (Figure 1c).

\subsection{Microbial assemblages and diversity}

Separate bacterial and fungal community profiling was conducted for 24 bulk soil and 24 rhizosphere samples to assess the structural and functional differences of soil microbial communities between CCS and FS. Briefly, bacterial community profiling yielded a total of 1353973 sequences, ranging from 35649 to 74817 . Fungal profiling resulted in 1653670 sequences, ranging from 38903 and 74 939. After subsampling of each sample to 11000 (bacterial) and 10000 (fungi) sequences (Figure S1), 3921 bacterial (Table S1) and 1099 fungal (Table S2) OTUs were identified across all samples.

Although no significant differences were found between C_NS and F_NS (neither in bacterial community richness nor in fungal community richness), alpha-diversity indexes indicated that bacterial community richness of bulk soil was higher in F_NS than in F_S; however, the differences were not significant for rhizosphere samples (Figure S2). F_NS had more fungal OTUs than F_S both in bulk soil and rhizosphere. However, the differences of bacterial or fungal community richness between C_NS and C_S were not pronounced neither in the bulk soil nor in the rhizosphere.

The taxonomy of microbial diversity was assessed by Circos. With regard to the abundance of bacterial phyla (Figure 2a), the rhizosphere and the bulk soil of N. tabacum were dominated by Proteobacteria, Actinobacteria, Bacteroidetes, Chloroflexi, Patescibateria, Firmicutes, Acidobacteria and Gemmatimonadetes. Proteobacteria, Actinobacteria, and Bacteroidetes always dominated both sample types, while Firmicutes was relatively more abundant in CCS than in FS. Moreover, soil sterilization treatment prior to sowing significantly decreased the abundances of Chloroflexi and Acidobacteria. Cyanobateria were only detected in rhizosphere samples (Figure 2b). With regard to the abundance of fungal classes (Figure S3), the rhizosphere of N. tabacum and bulk soil were mainly dominated by Sordariomycetes, Eurotiomycetes, Mortierellomycetes and Dothideomycetes. Moreover, Agaricomycetes and Microbotryomycetes were only found in rhizosphere samples. Sordariomycetes dominated both sample types. By examining between-sample variation (i.e., beta-diversity and Bray-Curtis distances), PCoA (Figure 3) indicated marked differences among microbial communities of bulk soil and rhizosphere. The one exception was that no significant difference was found between fungal communities in the rhizosphere.

\subsection{Specific microbial clades in}

\section{bulk soil and rhizosphere}

The different clades from phylum to genus level in both the bulk soil and rhizosphere were visualized by LEfSe (Figure 4). Specifically, in the bulk soil (Figure 4a, Table 1), the LDA score (LDA score $>4.0, p<$ 0.05) identified phyla Firmicutes and Bacteroidetes, as well as class Actinobacteria (order Bifidobacteriales, family Bifidobacteriaceae and genus Bifidobacterium ) in C_NS, as well as class Alphaproteobacteria (genusSphingobium ) in F_NS as differentially abundant bacterial taxa. Moreover, as the same classes were found in the bulk soil: Actinobacteria (family Microbacteriaceae and genusMicrobacterium ) in C_NS and Alphaproteobacteria (order Sphingomonadales, families Sphingomonadaceae and Xanthobacteraceae) in F_NS were found to be significantly enrichment in the rhizosphere. Moreover (Figure 4b, Table 2), the LDA score (LDA score $>3.0, p<0.05$ ) indicated significant enrichment of C_NS for class Eurotiomycetes. The phylum 
Rozellomycota and class Sordariomycetes in F_NS were among the most differentially abundant fungal taxa in bulk soil samples. OTUs representing the fungal class Leotiomycetes were significantly enriched in C_NS while Tremellomycetes and Dothideomycetes were detected in F_NS rhizosphere.

In addition, specific microbial clades were also detected in the reassembled soil bacterial communities (Figure $4 \mathrm{c}$, Table S3). Briefly, the LDA score (LDA score $>4.0, p<0.05$ ) indicated that class Gammaproteobacteria (family Rhodanobacteraceae) and Bacteroidia dominated F_S, thus forming bulk-soil bacterial communities. In the rhizosphere, C_S was selectively enriched with class Oxyphotobacteria, Gammaproteobacteria (genus Lysobacter ) and phylum Patescibacteria, while F_S showed great abundance of phylum Actinobacteria. Importantly, the LDA score (LDA score $>3.0, p<0.05$ ) suggested that no fungal clade was significantly enriched among the reassembled fungal communities.

\subsection{Influences of continuous cropping and natural fallow practice on rhizosphere effect}

This study documented the core microbiomes in rhizosphere of $N$. tabacum (Table S4). From the total of 1790 clustered bacterial OTUs found in rhizosphere, 432 were consistently present among all samples. Those 432 OTUs, classified up to genus level (Figure S4) were mainly involved in Sphingomonas (7.71\%), Luteimonas $(6.16 \%)$, Rhodanobacter (5.43\%), and Arthrobacter (4.67\%). Moreover, the core of the fungal microbiome was composed of 78 OTUs, which represented $13.2 \%$ of the total OTUs. Fusarium (15.52\%),Penicillium $(9.2 \%)$, and Mortierella $(6.92 \%)$ were the most abundant genera (Figure S4).

In addition to the core microbiomes of $N$. tabacum found in rhizosphere, the specific differences of the rhizosphere effect were investigated. For $N$. tabacumplants grown in CCS, $519(18.1 \%)$ and $425(19 \%)$ bacterial OTUs were found only in the rhizosphere of C_NS and C_S, respectively (Table S5). Compared with the bulk soil, the LDA score (LDA score $>3.0, p<0.05$ ) indicated that the orders Chloroplast and Corynebacteriales, genusSphingopyxis in C_NS, as well as class Oxyphotobacteria, order Micrococcales and genus Sphingopyxis in C_S were found only in the rhizosphere. Moreover, 183 (33.6\%) fungal OTUs related to the order Saccharomycetales and family Cladosporiaceae were found only in rhizosphere samples compared with those in the bulk soil in C_NS (LDA score > 2.0,p<0.05). Moreover, $194(58.1 \%)$ fungal OTUs related to class Dothideomycetes and genusSporobolomyces were only detected in the rhizosphere of C_S (LDA score > 2.0, $p<0.05)$. For $N$. tabacum plants grown in FS, $403(19.8 \%)$ and $279(23.1 \%)$ bacterial OTUs were only found in rhizosphere in F_NS and F_S, respectively (Table S6). Compared with the bulk soil, the LDA score (LDA score $>3.0, p<0.05)$ indicated that phyla Cyanobacteria and Firmicutes, order Corynebacteriales, and genus Nocardioidesin F_NS as well as phyla Cyanobacteria and Actinobacteria, family Lachnospiraceae, and genus Bacillus in C_S were only found in rhizosphere samples. In addition, $192(28.5 \%)$ fungal OTUs related to orders Venturiales and Capnodiales, and family Sympoventuriaceae were only found in the rhizosphere when compared with those in the bulk soil in F_NS (LDA score $>3.0, p<0.05)$. Although $83(30.6 \%)$ fungal OTUs were found only in the rhizosphere of F_S, no species was indicated under such filter criteria (LDA score $>2.0, p<0.05)$.

\section{DISCUSSION}

Recent studies indicated that changes of soil microbial communities as a result of various agricultural management practices contribute to plant performance (Zhou et al., 2014; Gao et al., 2019). Organic farming (Reganold \& Wachter, 2016), no-tillage (Ashworth et al., 2017), and rotation (Liu et al., 2019) have attracted the most scientific attention in the field of microbial research, while natural fallow practice has not been investigated in detail to this regard. Importantly, natural fallow practice is widely used worldwide because of labor shortage and areas unsuitable for mechanic agriculture. Therefore, the present study investigated the microbial mechanisms underlying the different response of $N$. tabacum plants to changes of soil microbiomes induced by natural fallow and continuous $N$. tabacum cropping practices. Clearly, different environmental conditions impose different selection forces on plants (Anderson, 2016; Jansson \& Hofmockel, 2020), while biomass accumulation and allocation strategies may vary over time and across environments (Poorter et al., 2012). First, the effects of microorganisms on $N$. tabacum fitness were investigated by reassembling soil microbial communities. The results indicated that changes of soil microbiomes significantly decreased the 
total biomass accumulation, changed biomass allocation patterns, and altered the investment strategies in N. tabacum leaves grown in FS. However, no similar changes were observed in plants grown in CCS (Figures 1a and $\mathrm{b}$ ). This suggests that soil microorganisms influence plant performance related to the abiotic soil context (Lozano et al., 2017; Mapelli et al., 2018). The LMA level is a key trait to assess plant growth and an important indicator for plant strategies (Poorter et al., 2009). In the present study, LMA levels showed a plastic response to environmental differences and plants grown in FS preferred to invest more in leaves and acquired a high leaf density during the process of microbial community reassembly (Figure 1c). In general, herbaceous or woody plants with a slower relative growth ratio (RGR) have a higher LMA level (Poorter et al., 2009). Thus, this implies that $N$. tabacum growth may suffer more from negative feedback of the reassembling soil microbial communities in response to natural fallow practice than in response to continuous cropping practice. In summary, these results suggested that soil microorganisms of FS play a more important role in determining N. tabacumfitness than those of CCS. Considering that, in practice, continuousN. tabacum cropping does not exceed three years, the damage from the changes of soil microbiomes to $N$. tabacum growth may be exaggerated at least within the short length of continuous cropping.

Soil microorganisms regulate many ecosystem processes and play key roles in nutrient cycling (Bender et al., 2016). However, the community structure and function also appear to be readily influenced by agricultural management practices. For example, bacterial network properties under continuous $N$. tabacum cropping are more sensitive to soil variables (Chen et al., 2018), while the abundances of several beneficial fungal species increased in a continuous soybean cropping practice (Liu et al., 2019). The present study identified no significant difference of microbial community richness under both management practices. However, bacterial and fungal community richness was higher for bulk soil in F_NS than in F_S, while no significant differences were found between C_NS and C_S. This suggests that the abiotic context of FS exerts stronger effects on the reassembly of soil microbiomes (de Vries et al., 2012; Erlandson et al., 2018). Furthermore, the taxonomy of microbial diversity indicated differences related to the management practice (Figures 2 and S3). For example, although Proteobacteria, Actinobacteria, and Bacteroidetes dominated both sample types, Firmicutes showed a relatively higher abundance in CCS than in FS. Previous studies indicated that Firmicutes was closely related to organic management (Hartman et al., 2018) and functioned in response to receiving manure fertilizer (Hartmann et al., 2014). Hence, this implies that relatively lower abundance of Firmicutes OTUs in FS may result in a low nutrient utilization efficiency on the bacterial level. This indirectly influenced $N$. tabacum growth, and finally decreased biomass accumulation. Moreover, PCoA showed marked differences among fungal communities of bulk soil; however, there was no significant difference in the rhizosphere of fungal communities (Figure 3). This indicates that the rhizosphere serves as a carbon-rich niche for the establishment of microbial communities while bulk soil is rapidly depleted of carbon and other nutrients by heterotrophic microbes (Sasse et al., 2018). Thus, this further suggests that similar colonization and coexistence conditions for fungi may exist in rhizosphere of $N$. tabacum plants both under continuous cropping and natural fallow practice. The results of the present study are consistent with a previous study, showing that species richness was less variable in the responses to different cropping systems than species composition (Hartman et al., 2018).

In addition, this study highlighted the differences of context-specific microbial clades between CCS and FS (Figures 4a and b; Tables 1 and 2). Several bacterial groups, such as Firmicutes and Actinobacteria, only showed significant enrichment in the bulk soil and rhizosphere of C_NS. This suggested that these bacterial groups were associated with receiving both manure fertilizer (Hartmann et al., 2014 and 2018) and soil fertility (Mapelli et al., 2018). In parallel, several bacterial groups were also found to be exclusively enriched in F_NS. For example, previous studies implied that Sphingomonadales (including family Sphingomonadaceae) has the ability to degrade a wide range of aromatic compounds (Balkwill et al., 2006), cope with stress conditions, and adapt to new habitats (Vaz-Moreira et al., 2011). Therefore, it has been suggested that the relatively high abundance of Sphingomonadales found in FS may be useful to improve $N$. tabacum growth environments. Several key microbes involved in Xanthobacteraceae contribute to nitrogen cycling (Zhu et al., 2018). The present study showed that Xanthobacteraceae were significantly enriched in rhizosphere in F_NS, suggesting that these microorganisms in the rhizosphere may play a key role in helping $N$. tabacum plants to 
acquire nitrogen nutrition when they grow in FS. In addition to bacterial clades, several fungal clades, such as Rozellomycota, Eurotiomycetes, and Sordariomycetes, were exclusively detected in the bulk soil in F_NS. This suggests that these play distinct roles in the plant-soil feedback when $N$. tabacumplants are grown in FS. The fungal class Leotiomycetes (family Myxotrichaceae and genus Oidiodendron ) was significantly enriched in rhizosphere in C_NS. Myxotrichaceae is cellulolytic, and was implied to play a significant role in the decomposition dynamics of litter (Rice et al., 2006). Tremellomycetes and Dothideomycetes dominated the rhizosphere in F_NS and may result in the difference between the structural and functional differences from C_NS. Moreover, this study also documented specific microbial clades in the reassembling soil microbial communities (Figure 4c, Table S3). For example, the bacterial family Rhodanobacteraceae, which belongs to the class of Gammaproteobacteria, dominated the rhizosphere in F_S, and was implied to have the capacity to degrade polycyclic aromatic hydrocarbons (Cazals et al., 2019). Importantly, no fungal clade was significantly enriched among the reassembled fungal communities. This again suggests that similar colonization and coexistence conditions may exist for fungi in the rhizosphere of $N$. tabacum . In conclusion, these differences in composition suggest that not only plant performance (Sasse et al., 2018), but also the specific differences in the abiotic context exert distinct effects on the reassembly of the soil microbiome.

As far as we know, several common factors, including abiotic and biotic factors, likely lead to the assembly of a core microbiome (Naylor et al., 2017; Erlandson et al., 2018; Lundberg \& Teixeira, 2018; Perez-Jaramillo et al., 2019). In the present study, only $24.1 \%$ bacterial OTUs and $13.2 \%$ fungal OTUs were identified as the core microbiomes (Table S4), suggesting that most OTUs were specific and that $N$. tabacum plants may have a strong strength of rhizosphere effect. Previous studies suggested that the strength of the rhizosphere effect is likely associated with the developmental stage of a plant (Chaparro et al., 2013), root exudation (Zhalnina et al., 2018), host genotype (Bulgarelli et al., 2015), and domestication (Edwards et al., 2019). This study further compared the compositional differences between bulk soil and rhizosphere and identified several context-specific microbial species, especially those exclusively enriched in the rhizosphere (Tables S5 and S6). This suggested that those species likely elucidate the differences of rhizosphere bacterial and fungal microbiomes between FS and CCS.

\section{CONCLUSIONS}

The findings of the present study imply that specific microbial community assembly and especially a number of context-specific microbial clades under different management practices (continuous cropping and natural fallow) may lead to different growth and development in $N$. tabacum. In addition, a set of positive changes of soil microbiomes induced by the natural fallow practice could not completely enhance $N$. tabacum fitness, while additional agricultural management practices such as targeted microbial fertilizer application or rotation may compensate for the shortcomings arising from a single measure.

\section{ACKNOWLEDGEMENTS}

This research was supported by the scientific research projects of the Sichuan Branch of the China National Tobacco Corporation (No. 201851080024069).

\section{CONFLICT OF INTEREST}

All authors declare that they have no conflict of interest.

\section{SUPPLEMENTARY INFORMATION}

Supplementary FIGURE S1 Rarefaction and Goods's coverage index. Rarefactions were assessed and all samples had a coverage of more than 90\% (Subsampling: bacteria (11 000 reads): > 93\%; fungi (10 000 reads): $>98 \%$ ).

Supplementary FIGURE S2 Alpha-diversity indexes of Sobs and Ace for bulk soil and rhizosphere (a, b) bacterial and (c, d) fungal communities of CCS and FS, respectively. Significance values of the Welch's t-test for the effects are indicated as follows: ${ }^{*}, p<0.05 ;{ }^{* *}, p<0.01$. 
Supplementary FIGURE S3 Overview of the taxonomy of fungal diversity on class level in (a) bulk soil and (b) rhizosphere of CCS and FS.

Supplementary FIGURE S4 Pie plots show the core microbiomes in rhizosphere of tobacco. Bacterial and fungal OTUs were classified up to genus level, respectively. p: phylum; c: class; o: order; f: family; g: genus.

Supplementary TABLE S1Taxon analysis on bacterial communities of 24 samples. C_NS: continuous cropping soil without sterilization treatment; C_S: continuous cropping soil with sterilization treatment; F_NS: natural fallow soil without sterilization treatment; and F_S: natural fallow soil with sterilization treatment.

Supplementary TABLE S2 Taxon analysis on fungal communities of 24 samples.

Supplementary TABLE S3 Linear discriminant analysis (LDA score $>4.0, p<0.05$ ) effect size (LEfSe) of bacterial species in bulk soil and rhizosphere in the reassembled soil microbial communities of CCS and FS.

Supplementary TABLE S4 The core microbiomes in rhizosphere of tobacco found in continuous cropping soil (CCS) and natural fallow soil (FS).

Supplementary TABLE S5Effects of continuous tobacco cropping practice on rhizosphere effect. Microbial species exclusively enriched in bulk soil or rhizosphere. Species numbers over $20 \%$ of the total numbers were indicated in bold. "/": total species numbers less than 10 were not counted.

Supplementary TABLE S6 Effects of natural fallow practice on rhizosphere effect. Microbial species exclusively enriched in bulk soil or rhizosphere. Species numbers over $20 \%$ of the total numbers were indicated in bold. "/": total species numbers less than 10 were not counted.

\section{REFERENCES}

Adams, R.I., Miletto, M., Taylor, J.W., \& Bruns, T.D. (2013). Dispersal in microbes: fungi in indoor air are dominated by outdoor air and show dispersal limitation at short distances. The ISME Journal , 7, 1262-1273.

Anderson, J.T. (2016). Plant fitness in a rapidly changing world.New Phytologist, 210, 81-87.

Ashworth, A.J., DeBruyn, J.M., Allen, F.L., Radosevich, M., \& Owens, P.R. (2017). Microbial community structure is affected by cropping sequences and poultry litter under long-term no-tillage.Soil Biology and Biochemistry , 114, 210-219.

Balkwill, D.L., Fredrickson, J.K., \& Romine, M.F. (2006). Sphingomonas and related genera. In: Dworkin M, Falkow S, Rosenberg E, Schleifer KH, Stackebrandt E (Eds.), The Prokaryotes. Springer-Verlag, New York, pp 605-629.

Bender, S.F., Wagg, C., \& van der Heijden, M.G.A. (2016). An underground revolution: biodiversity and soil ecological engineering for agricultural sustainability. Trends in Ecology and Evolution, 31, 440-452.

Berendsen, R.L., Pieterse, C.M., \& Bakker, P.A. (2012). The rhizosphere microbiome and plant health. Trends in Plant Science, 17, 478-486.

Berg, G. (2009). Plant-microbe interactions promoting plant growth and health: perspectives for controlled use of microorganisms in agriculture. Applied Microbiology and Biotechnology, 84, 11-18.

Beulig, F., Urich, T., Nowak, M., Trumbore, S.E., Gleixner, G., Gilfillan, G.D., Fjelland, K.D., \& Küsel, K. (2016). Altered carbon turnover processes and microbiomes in soils under long-term extremely high CO2 exposure. Nature Microbiology, 1, 15025.

Bolger, A.M., Lohse, M., \& Usadel, B. (2014). Trimmomatic: a flexible trimmer for Illumina sequence data. Bioinformatics , 30, 2114-2120. 
Bonanomi, G., Lorito, M., Vinale, F., \& Woo, S.L. (2018). Organic amendments, beneficial microbes, and soil microbiota: toward a unified framework for disease suppression. Annual Review of Phytopathology, 56, $1-20$.

Bulgarelli, D., Garrido-Oter, R., Münch, P.C., Weiman, A., Dröge, J., Pan, Y., McHardy, A.C., \& SchulzeLefert, P. (2015). Structure and function of the bacterial root microbiota in wild and domesticated barley. Cell Host and Microbe, 17, 392-403.

Caporaso, J.G., Kuczynski, J., Stombaugh, J., Bittinger, K., Bushman, F.D., Costello, E.K., .. Knight, R. (2010). QIIME allows analysis of high-throughput community sequencing data. Nature Methods , 7, 335-336.

Carrion, V.J. (2019). Deciphering rhizosphere microbiome assembly of wild and modern common bean (Phaseolus vulgaris ) in native and agricultural soils from Colombia. Microbiome , 7, 114.

Cazals, F., Huguenot, D., Crampon, M., Colombano, S., Betelu, S., Galopin, N., .. Rossano, S. (2019). Production of biosurfactant using the endemic bacterial community of a PAHs contaminated soil, and its potential use for PAHs remobilization. Science of the Total Environment, 709, 136143.

Chaparro, J.M., Badri, D.V., \& Vivanco, J.M. (2013). Rhizosphere microbiome assemblage is affected by plant development. The ISME Journal , 8, 790-803.

Chen, S., Qi, G., Luo, T., Zhang, H., Jiang, Q., Wang, R., Zhao, X. (2018). Continuous-cropping tobacco caused variance of chemical properties and structure of bacterial network in soils. Land Degradation and Development , 29, 4106-4120.

De Vries, F.T., Manning, P., Tallowin, J.R.B., Mortimer, S.R., Pilgrim, E.S., Harrison, K.A. .. Bardgett, R.D. (2012). Abiotic drivers and plant traits explain landscape-scale patterns in soil microbial communities. Ecology Letters , 15, 1230-1239.

Duran, P., Thiergart, T., Garrido-Oter, R., Agler, M., Kemen, E., Schulze-Lefert, P., \& Hacquard, S. (2018). Microbial interkingdom interactions in roots promote Arabidopsis survival. Cell , 175, 973-983.

Edgar, R.C. (2013). UPARSE: highly accurate OTU sequences from microbial amplicon reads.Nature Methods , 10, 996-998.

Edwards, J., Santos-Medellin, C., Nguyen, B., Kilmer, J., Liechty, Z., Veliz, E., Ni, J., Philips, G., \& Sundaresan, V. (2019). Soil domestication by rice cultivation results in plant-soil feedback through shifts in soil microbiota. Genome Biology , 20, 221.

Erlandson, S., Wei, X., Savage, J., Cavender-Bares, J., \& Peay, K. (2018). Soil abiotic variables are more important than Salicaceae phylogeny or habitat specialization in determining soil microbial community structure. Molecular Ecology , 27, 2007-2024.

Gao, Z., Han, M., Hu, Y., Li, Z., Liu, C., Wang, X., ... Ma, Z. (2019). Effects of continuous cropping of sweet potato on the fungal community structure in rhizospheric soil. Frontiers in Microbiology , 10, 2269.

Garbeva, P., van Veen, J.A., \& van Elsas, J.D. (2004). Microbial diversity in soil: selection of microbial populations by plant and soil type and implications for disease suppressiveness. Annual Review of Phytopathology , 42, 243-270.

Hartman, K., van der Heijden, M.G.A., Wittwer, R.A., Banerjee, S., Walser, J.C., \& Schlaeppi, K. (2018). Cropping practices manipulate abundance patterns of root and soil microbiome members paving the way to smart farming. Microbiome, 6, 14 .

Hartmann, M., Frey, B., Mayer, J., Mäder, P., \& Widmer, F. (2014). Distinct soil microbial diversity under long-term organic and conventional farming. The ISME Journal , 9, 1177-1194.

Hu, L., Robert, C.A.M., Cadot, S., Zhang, X., Ye, M., Li, B., .. Erb, M. (2018). Root exudate metabolites 
drive plant-soil feedbacks on growth and defense by shaping the rhizosphere microbiota. Nature Communications , 9, 2738 .

Jansson, J.K., \& Hofmockel, K.S. (2020). Soil microbiomes and climate change. Nature Reviews Microbiology , 18, 35-46.

Krzywinski, M.I., Schein, J.E., Birol, I., Connors, J., Gascoyne, R., Horsman, D., Jones, S.J., \& Marra, M.A. (2009). Circos: An information aesthetic for comparative genomics. Genome Research , 19, 1639-1645

Kuypers, M.M.M., Marchant, H.K., \& Kartal, B. (2018). The microbial nitrogen-cycling network. Nature Review Microbiology , 16, 263-276.

Kwak, M.J., Kong, H.G., Choi, K., Kwon, S.K., Song, J.Y., Lee, J., .. Kim, J.F. (2018). Rhizosphere microbiome structure alters to enable wilt resistance in tomato. Nature Biotechnology , 36, 1100-1109.

Lau, J.A., \& Lennon, J.T. (2012). Rapid responses of soil microorganisms improve plant fitness in novel environments.Proceedings of the National Academy of Sciences of the United States of America , 109, 1405814062 .

Liu, J., Yao, Q., Li, Y., Zhang, W., Mi, G., Chen, X., Yu, Z., \& Wang, G. (2019). Continuous cropping of soybean alters the bulk and rhizospheric soil fungal communities in a Mollisol of Northeast PR China. Land Degradation and Development, 30, 1725-1738.

Lozano, Y.M., Armas, C., Hortal, S., Casanoves, F., \& Pugnaire, F.I. (2017). Disentangling above- and below-ground facilitation drivers in arid environments: the role of soil microorganisms, soil properties and microhabitat. New Phytologist, 216, 1236-1246.

Lundberg, D.S., \& Teixeira, P. (2018). Root-exuded coumarin shapes the root microbiome. Proceedings of the National Academy of Sciences of the United States of America, 115, 5629-5631.

Luo, S., Schmid, B., De Deyn, G.B., Yu, S., \& Field, K. (2018). Soil microbes promote complementarity effects among co-existing trees through soil nitrogen partitioning. Functional Ecology, 32, 1879-1889.

Magoc, T., \& Salzberg, S.L. (2011). FLASH: fast length adjustment of short reads to improve genome assemblies. Bioinformatics , 27, 2957-2963.

Mapelli, F., Marasco, R., Fusi, M., Scaglia, B., Tsiamis, G., Rolli, E., .. Daffonchio, D. (2018). The stage of soil development modulates rhizosphere effect along a High Arctic desert chronosequence. The ISME Journal , 12, 1188-1198.

Mori, H., Maruyama, F., Kato, H., Toyoda, A., Dozono, A., Ohtsubo, Y., .. Kurokawa, K. (2014). Design and experimental application of a novel non-degenerate universal primer set that amplifies prokaryotic $16 \mathrm{~S}$ rRNA genes with a low possibility to amplify eukaryotic rRNA Genes.DNA Research , 21, 217-227.

Naylor, D., DeGraaf, S., Purdom, E., \& Coleman-Derr, D. (2017). Drought and host selection influence bacterial community dynamics in the grass root microbiome. The ISME Journal , 11, 2691-2704.

Ortas, I. (1997). Determination of the extent of rhizosphere soil. Communications in Soil Science and Plant Analysis , 28, 1767-1776.

Poorter, H., Niinemets, Ü., Poorter, L., Wright, I.J., \& Villar, R. (2009). Causes and consequences of variation in leaf mass per area (LMA): a meta-analysis. New Phytologist , 182, 565-588.

Poorter, H., Niklas, K.J., Reich, P.B., Oleksyn, J., Poot, P., \& Mommer, L. (2012). Biomass allocation to leaves, stems and roots: meta-analyses of interspecific variation and environmental control.New Phytologist , 193, 30-50.

Quast, D., Pruesse, E., Yilmaz, P., Gerken, J., Schweer, T., Yarza, P. Peplies, J., \& Glöckner, F.O. (2013). The SILVA ribosomal RNA gene database project: improved data processing and web-based tools. Nucleic Acids Research , 41, D590-D596. 
Reganold, J.P., \& Wachter, J.M. (2016). Organic agriculture in the twenty-first century. Nature Plants , 2, 15221.

Rice, A.V., Tsuneda, A., \& Currah, R.S. (2006). In vitro decomposition of Sphagnum by some microfungi resembles white rot of wood.FEMS Microbiology Ecology , 56, 372-382.

Sasse, J., Martinoia, E., \& Northen, T. (2018). Feed your friends: do plant exudates shape the root microbiome? Trends in Plant Science, 23, 25-41.

Segata, N., Izard, J., Waldron, L., Gevers, D., Miropolsky, L., Garrett, W.S., \& Huttenhower, C. (2011). Metagenomic biomarker discovery and explanation. Genome Biology, 12, R60.

Teste, F.P., Kardol, P., Turner, B.L., Wardle, D.A., Zemunik, G., Renton, M., \& Laliberté, E. (2017). Plantsoil feedback and the maintenance of diversity in Mediterranean-climate shrublands.Science, 355, 173-176.

Vaz-Moreira, I., Nunes, O.C., \& Manaia, C.M. (2011). Diversity and antibiotic resistance patterns of Sphingomonadaceae isolates from drinking water. Applied and Environmental Microbiology , 77, 5697-5706.

Venkatachalam, L., Gopinath, S., \& Bais, H.P. (2014). Functional soil microbiome: belowground solutions to an aboveground problem. Plant Physiology , 166, 689-700.

Wang, Y., Li, C., Tu, C., Hoyt, G., DeForest, J., \& Hu, S. (2017). Long-term no-tillage and organic input management enhanced the diversity and stability of soil microbial community. Science of the Total Environment , 609, 341-347.

Xun, W., Yan, R., Ren, Y., Jin, D., Xiong, W., Zhang, G., .. Zhang, R. (2018). Grazing-induced microbiome alterations drive soil organic carbon turnover and productivity in meadow steppe.Microbiome , 6, 170.

Zhalnina, K., Louie, K.B., Hao, Z., Mansoori, N., da Rocha, U.N., Shi, S., ...Brodie, E.L. (2018). Dynamic root exudate chemistry and microbial substrate preferences drive patterns in rhizosphere microbial community assembly. Nature Microbiology , 3, 470-480.

Zhou, J.Z., Xue, K., Xie, J.P., Deng, Y., \& Luo, Y. (2012). Microbial mediation of carbon-cycle feedbacks to climate warming. Nature Climate Change, 2, 106-110.

Zhou, X., Gao, D., Liu, J., Qiao, P., Zhou, X., Lu, H., .. Wu, F. (2014). Changes in rhizosphere soil microbial communities in a continuously monocropped cucumber (Cucumis sativus L.) system.European Journal of Soil Biology , 60, 1-8.

Zhu, B.K., Fang, Y.M., Zhu, D., Christie, P., Ke, X., \& Zhu, Y.G. (2018). Exposure to nanoplastics disturbs the gut microbiome in the soil oligochaete Enchytraeus crypticus . Environmental Pollution, 239, 408-415.

TABLE 1 Linear discriminant analysis (LDA score $>4.0, p<0.05$ ) effect size (LEfSe) of bacterial species in bulk soil and rhizosphere of CCS and FS.

\begin{tabular}{lllll}
\hline Species name & Group & Mean & LDA_value & $p$ value \\
\hline genus: Bifidobacterium & Bulk soil C_NS & 4.37 & 4.08 & 0.024 \\
genus: Lactobacillus & Bulk soil C_NS & 4.37 & 4.07 & 0.024 \\
class: Clostridia & Bulk soil C_NS & 4.95 & 4.63 & 0.04 \\
order: Bacteroidales & Bulk soil C_NS & 4.63 & 4.3 & 0.038 \\
order: Lactobacillales & Bulk soil C_NS & 4.56 & 4.24 & 0.025 \\
family: Lactobacillaceae & Bulk soil C_NS & 4.4 & 4.08 & 0.024 \\
class: Bacilli & Bulk soil C_NS & 4.69 & 4.36 & 0.025 \\
order: Bifidobacteriales & Bulk soil C_NS & 4.37 & 4.04 & 0.028 \\
family: Bifidobacteriaceae & Bulk soil C_NS & 4.37 & 4.06 & 0.028 \\
order: Clostridiales & Bulk soil C_NS & 4.95 & 4.61 & 0.04 \\
family: Lachnospiraceae & Bulk soil C_NS & 4.57 & 4.26 & 0.022
\end{tabular}




\begin{tabular}{lllll}
\hline Species name & Group & Mean & LDA_value & $p$ value \\
\hline family: Ruminococcaceae & Bulk soil C_NS & 4.61 & 4.3 & 0.023 \\
phylum: Firmicutes & Bulk soil C_NS & 5.17 & 4.82 & 0.025 \\
genus: Sphingomonas & Bulk soil F_NS & 5.23 & 4.76 & 0.034 \\
family: Microbacteriaceae & Rhizosphere C_NS & 4.53 & 4.15 & 0.048 \\
family: Xanthomonadaceae & Rhizosphere C_NS & 4.96 & 4.5 & 0.044 \\
genus: Microbacterium & Rhizosphere C_NS & 4.36 & 4.07 & 0.026 \\
family: Sphingomonadaceae & Rhizosphere F_NS & 5.33 & 4.84 & 0.038 \\
order: Sphingomonadales & Rhizosphere F_NS & 5.33 & 4.79 & 0.038 \\
family: Xanthobacteraceae & Rhizosphere F_NS & 4.53 & 4.05 & 0.038 \\
class: Alphaproteobacteria & Rhizosphere F_NS & 5.51 & 4.91 & 0.038 \\
\hline
\end{tabular}

TABLE 2 Linear discriminant analysis (LDA score $>3.0, p<0.05$ ) effect size (LEfSe) of fungal species in bulk soil and rhizosphere of CCS and FS.

\begin{tabular}{lllll}
\hline Species name & Group & Mean & LDA_value & $p$ value \\
\hline family: Herpotrichiellaceae & Bulk soil C_NS & 3.29 & 3.01 & 0.034 \\
genus: Penicillium & Bulk soil C_NS & 5.02 & 4.63 & 0.038 \\
genus: Cylindrocarpon & Bulk soil F_NS & 3.26 & 3 & 0.035 \\
family: Clavicipitaceae & Bulk soil F_NS & 3.43 & 3.11 & 0.033 \\
phylum: Rozellomycota & Bulk soil F_NS & 3.74 & 3.43 & 0.015 \\
genus: Metarhizium & Bulk soil F_NS & 3.42 & 3.07 & 0.032 \\
genus: Oidiodendron & Rhizosphere C_NS & 3.4 & 3.1 & 0.023 \\
family: Myxotrichaceae & Rhizosphere C_NS & 3.4 & 3.05 & 0.023 \\
order: Venturiales & Rhizosphere F_NS & 2 & 3.15 & 0.013 \\
family: Trimorphomycetaceae & Rhizosphere F_NS & 3.75 & 3.41 & 0.038 \\
genus: Saitozyma & Rhizosphere F_NS & 3.75 & 3.4 & 0.038 \\
family: Phaeosphaeriaceae. & Rhizosphere F_NS & 3.49 & 3.11 & 0.03 \\
\hline
\end{tabular}

\section{FIGURE LEGENDS}

FIGURE 1Effects of soil microorganisms on plant fitness (mean $\pm \mathrm{SE}, \mathrm{n}=3$ ). The following metrics were used for this comparison: (a) biomass accumulation, (b) allocation patterns, and (c) leaf dry mass per unit area (LMA) of $N$. tabacum grown in continuous cropping soil (CCS) and natural fallow soil (FS). Significance values of the factorial analysis (ANOVA) for the effects are indicated as follows: ${ }^{*}, p<0.05 ;{ }^{* *}, p<0.01$. Filled (or open) points show soil without (or with) sterilization treatment.

FIGURE 2Overview of the taxonomy of bacterial diversity on phylum level in (a) bulk soil and (b) rhizosphere of CCS and FS.

FIGURE 3 Principal coordinate analysis (PCoA) using Bray-Curtis distance between samples for taxonomic profiles of bacteria and fungi (operational taxonomic unit (OUT) level for the 16S rRNA data set) for bulk soils and rhizosphere of CCS and FS. Permutational multivariate analysis of variance (PERMANOVA) was used to assess group differences.

FIGURE 4 Significantly retrieved discriminant taxa for bulk soil and rhizosphere bacterial and fungi communities of CCS and FS. The cladogram provides a taxonomic representation of statistically consistent differences between rhizosphere and bulk soil for (a) bacterial communities, (b) fungal communities, and (c) bacterial communities of the reassembled soil microbial communities. The tables below each cladogram report phyla/classes that statistically significantly discriminated bulk soilvs rhizosphere. Filter criteria: for bacterial microbiomes: LDA score $>4.0, p<0.05$; for fungal microbiomes: LDA score $>3.0, p<0.05$. 

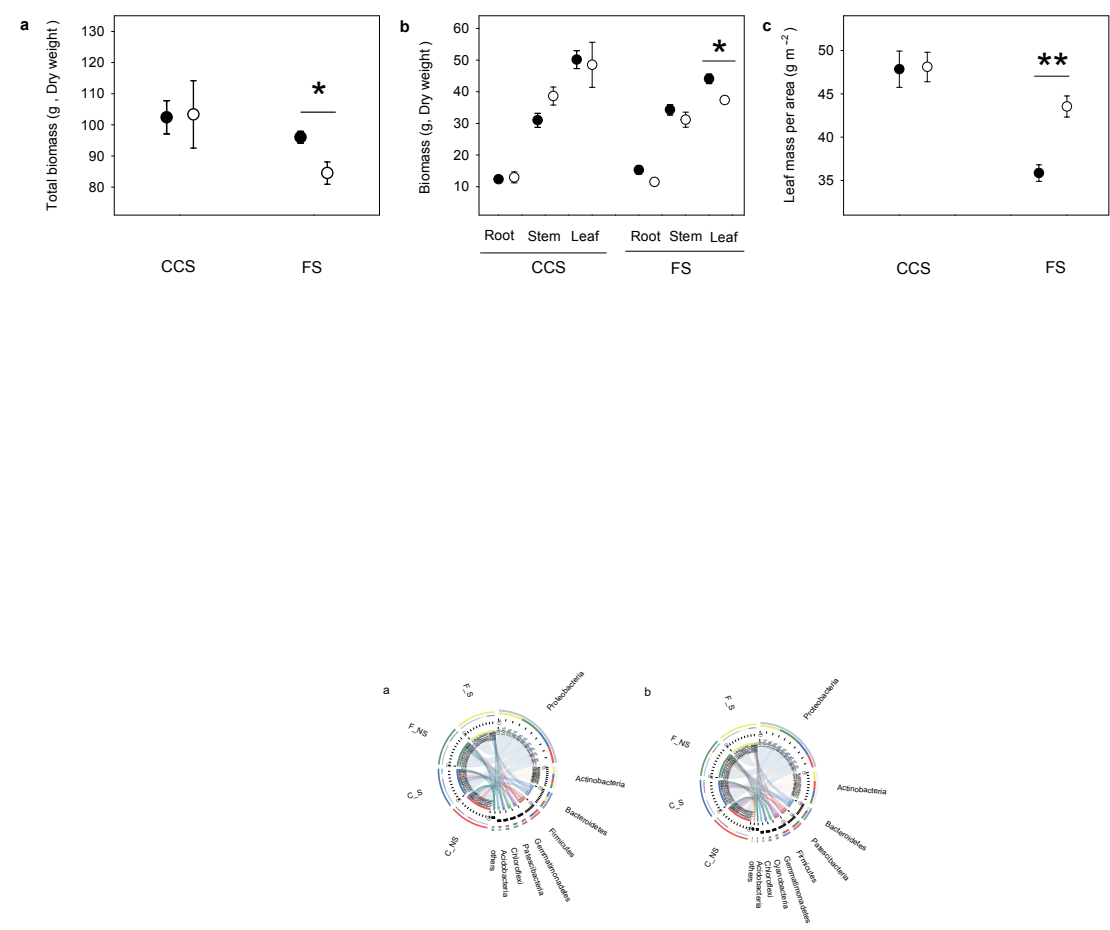

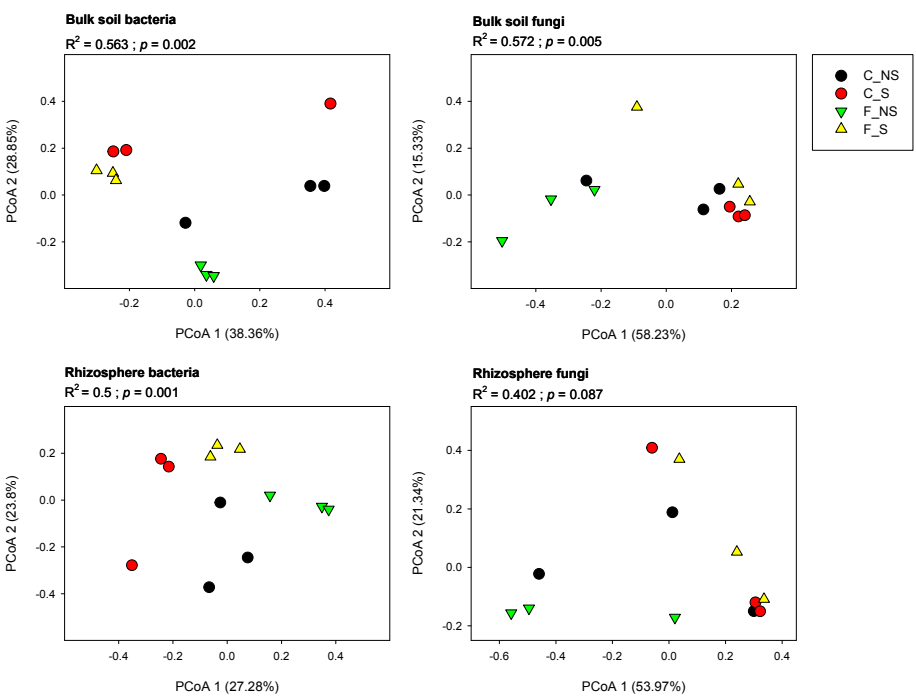


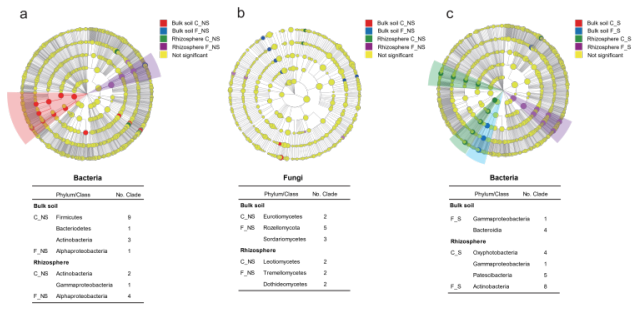

